\title{
CAEP 2018 Academic Symposium: Recommendations for developing and supporting Global Emergency Medicine in Canadian academic emergency departments and divisions
}

\author{
Amanda Collier, MD, DTMH*; Gregory Marton, $\mathrm{MD}^{\dagger}$; Shannon Chun, $\mathrm{MD}^{\ddagger}$; \\ Cheri Nijssen-Jordan, MD, MBA ${ }^{\S}$; Susan A. Bartels, MD, $\mathrm{MPH}^{*}$; Simon Pulfrey, $\mathrm{MD}^{* *}$; \\ Eddy Lang, MDI; Michael Schull, MD, MSc ${ }^{\dagger+}$; Megan Landes, MD, MSc ${ }^{\ddagger \neq}$; \\ Kirsten Johnson, MD, MPH ${ }^{\S \S}$
}

\section{ABSTRACT}

Objectives: The objective of the CAEP Global Emergency Medicine (EM) panel was to identify successes, challenges, and barriers to engaging in global health in Canadian academic emergency departments, formulate recommendations for increasing engagement of faculty, and guide departments in developing a Global EM program.

Methods: A panel of academic Global EM practitioners and residents met regularly via teleconference in the year leading up to the CAEP 2018 Academic Symposium. Recommendations were drafted based on a literature review, three mixed methods surveys (CAEP general members, Canadian Global EM practitioners, and Canadian academic emergency department leaders), and panel members' experience. Recommendations were presented at the CAEP 2018 Academic Symposium in Calgary and further refined based on feedback from the Academic Section.

Results: A total of nine recommendations are presented here. Seven of these are directed towards Canadian academic departments and divisions and intend to increase their engagement in Global EM by recognizing it as an integral part of the practice of emergency medicine, deliberately incorporating it into strategic plans, identifying local leaders, providing tangible supports (i.e., research, administration or financial support, shift flexibility), mitigating barriers, encouraging collaboration, and promoting academic deliverables. The final two recommendations pertain to CAEP increasing its own engagement and support of Global EM.
Conclusions: These recommendations serve as guidance for Canadian academic emergency departments and divisions to increase their engagement in Global EM.

Keywords: Global Emergency Medicine, International

Emergency Medicine, global health, academic engagement

\section{INTRODUCTION}

As Canadian emergency departments strive for excellence in providing emergency care, education, and research, one consideration should be sharing that expertise in the global setting. The burden of death and morbidity for emergency patients in low- and middle-income countries (LMICs) is estimated as 120 times higher than for patients in North American emergency departments. ${ }^{1}$ Aligned with the International Federation for Emergency Medicine (IFEM) principle that all countries should provide unrestricted access to emergency healthcare, ${ }^{2}$ there is continued need for global development of emergency care and an opportunity for Canadian physicians to contribute. Emergency medicine (EM) physicians also have an ideal skill set to be effective in humanitarian response or disaster relief. ${ }^{3}$ With increasing emergency visits by refugees, visitors, and

From the *Department of Emergency Medicine, and Public Health Sciences, Queen's University, Kingston, ON; †Department of Emergency Medicine, McGill University, Montreal, QC; $¥$ Department of Medicine, University of Toronto, Toronto, ON; Departments of §Pediatrics and $\mathbb{\text { IEmergency }}$ Medicine, University of Calgary, Calgary, AB; **Department of Emergency Medicine, University of British Columbia, Vancouver, BC; $\uparrow+S u n n y b r o o k$ Health Sciences Centre, University of Toronto, Toronto, ON; and; Departments of ¥¥Departments of Family and Community Medicine, University of Toronto, Toronto, ON; and the §§Department of Emergency Medicine, McGill University, Montreal, QC.

Correspondence to: Dr. Amanda Collier, Department of Emergency Medicine, Queen's University, 76 Stuart Street, Kingston, ON K7L 2V7; Email: ac219@queensu.ca

(C) Canadian Association of Emergency Physicians 
returning travellers, ${ }^{4,5}$ some elements of Global EM competence are required of all emergency physicians, even if they do not practise outside of their local environment.

Currently, there is little recognition of Global EM as an academic niche in Canada. Although many Canadian EM physicians work internationally, it is usually arranged independently, outside of their academic commitments. ${ }^{6-8}$ In contrast, there are many global/international EM divisions within academic departments in the United States and over 40 global/international EM fellowships in U.S. medical schools, highlighting the rapid growth of this area. ${ }^{9,10}$ The availability of international opportunities has been shown to positively influence resident rankings of prospective academic programs. ${ }^{11}$

The Canadian Association of Emergency Physicians' (CAEP) Academic Section aims to improve emergency care by supporting education scholarship, research, and leadership/administration in EM, including delivering an annual Academic Symposium. ${ }^{12}$ For the 2018 Leadership Symposium, a Global EM panel was formed to identify successes and barriers to engaging in Global EM practice, and develop recommendations to increase engagement in Canadian academic departments and divisions. For the purposes of the Global EM panel, the broad consensus definition of Global EM developed by the Global Emergency Medicine Literature Review was used. ${ }^{13}$

\section{METHODS}

The CAEP Global EM panel consisted of 10 practising physicians and two EM residents with expertise in global health practice, education, and research. The panel met twice by teleconference in November 2017 to define the study question and methods, and monthly thereafter to develop recommendations. A mixed methods approach was used, including a review of the literature, surveys of CAEP members, Global EM practitioners, and Canadian EM leaders, and input from the professional experience of panel members.

The primary goal of the panel was to address the question: What is required to develop and support global bealth engagement in Canadian academic emergency departments? Our objectives were to 1) explore experiences in Global EM in Canada, including successes, challenges, and barriers to global health practice; 2 ) describe ingredients for successful engagement within the following areas: academic environment, leadership, faculty needs; 3 ) develop key recommendations for global health engagement in academic departments; and 4) highlight special considerations and future areas for exploration in enhancing Global EM practice in Canadian academic emergency departments/divisions.

\section{Literature review}

A literature search of Medline, Embase, and Global Health databases was performed from inception to March 17, 2018, using the search terms emergency medicine, global health, international educational exchange, international cooperation, international education, and medical missions. Abstracts were reviewed by two panel members (AC, SB) and classified into categories (Supplemental Figure 1). Disagreements were resolved by consensus. The most recent 5 years of relevant EM journal table of contents (CFEM, Annals of Emergency Medicine, Academic Emergency Medicine, Emergency Medicine fournal) and the websites of Canadian EM academic departments/divisions were also searched for additional articles.

\section{Quantitative and qualitative surveys}

Three mixed methods surveys were designed for CAEP general members, Canadian Global EM practitioners, and Canadian academic emergency department leaders (using a CAEP leadership forum list). Surveys were distributed electronically in March and April 2018, and followed by three reminder emails. Quantitative surveys were designed using Survey Monkey and analysed using Excel.

\section{RESULTS}

\section{Literature review}

The search strategy returned 376 articles with 1 additional article identified by hand searching recent EM journals. Although many articles described aspects of practising global EM (see Supplemental Figure 1), only three specifically described methods of an academic unit engaging in Global EM. ${ }^{14-16}$ Collectively, these three articles addressed the role of clinician educator/ researcher, academic productivity, training/fellowships, and academic collaborations with LMIC universities or local/national/international organizations.

\section{CAEP general members survey}

Emails were sent to 1,280 members with 144 responses (11.3\%). Responses are shown in Table 1. Sixty percent of respondents were engaged in global health and $44 \%$ 
engaged once a year or more; $70 \%$ would like to be more engaged than their current level. Regarding the role of Global EM in Canada, the top three responses were that Global EM should be "encouraged with protected time and funds," "supported but not as its own subspecialty," and "recognized as a subspecialty." Commonly identified barriers were a lack of protected time, family/personal obligations, and clinical/departmental obligations. Tangibles (administrative support, academic recognition, shift flexibility, covered expenditures), financial support, and protected time were identified as being most important for supporting Global EM.

\section{Canadian Global EM practitioner surveys}

A total of 27 EM practitioners with global health experience were contacted via email, with 6 respondents to the quantitative survey. The majority $(80 \%)$ dedicated less than $20 \%$ of their full-time equivalent (FTE) to Global EM activities. Again, lack of departmental support, lack of protected time, and financial constraints were identified as barriers. Most respondents agreed that increased shift flexibility and increased funding were important to improving Global EM engagement in Canada.

To expand on the quantitative survey, 34 Global EM practitioners were contacted for completion of a semistructured interview. Fifteen responded, describing Global EM activities in 26 different countries. Half of respondents reported doctorate or masters level training in relevant areas (public health, global health), whereas one-third reported no additional training. Similar barriers were again identified. This group particularly identified Global EM mentors as critical to career development. Respondents also acknowledged a lack of collaboration between Global EM practitioners and the need to produce academic deliverables in conjunction with their Global EM work.

\section{Canadian emergency medicine leaders survey}

Fifty Canadian academic emergency department leaders were contacted with seven responses (see Supplementary Material, Table 2). Eighty-six percent had no formalized Global EM support, yet most respondents (71\%) thought that Global EM should have protected time and funding. In addition to barriers previously discussed, leaders also identified a lack of academic recognition and a lack of departmental knowledge or expertise in Global EM. Fifty-seven percent believed there was a desire in their department to integrate Global EM activities.

\begin{tabular}{|c|c|}
\hline Demographics & n (\%) \\
\hline \multicolumn{2}{|l|}{ Sex } \\
\hline Male & $80(56 \%)$ \\
\hline Female & $63(44 \%)$ \\
\hline \multicolumn{2}{|l|}{ Years in practice } \\
\hline In residency & $13(9 \%)$ \\
\hline $1-5$ & $28(20 \%)$ \\
\hline $6-10$ & $14(10 \%)$ \\
\hline $11-15$ & $23(16 \%)$ \\
\hline $16-20$ & $13(9 \%)$ \\
\hline$>20$ & $52(36 \%)$ \\
\hline \multicolumn{2}{|l|}{ Training } \\
\hline FRCPC & $51(35 \%)$ \\
\hline CCFP & $66(46 \%)$ \\
\hline Other & $27(19 \%)$ \\
\hline \multicolumn{2}{|l|}{ Type of practice } \\
\hline Academic ED & $78(54 \%)$ \\
\hline Community ED & $44(31 \%)$ \\
\hline Mixed & $19(13 \%)$ \\
\hline \multicolumn{2}{|l|}{ Do you engage in Global EM activities?* } \\
\hline No & $42(40 \%)$ \\
\hline Yes, locally & $14(13 \%)$ \\
\hline Yes, internationally & $26(25 \%)$ \\
\hline Yes, both locally and internationally & $22(21 \%)$ \\
\hline \multicolumn{2}{|c|}{ How often do you engage in Global EM activities? } \\
\hline Not at all & $31(30 \%)$ \\
\hline Less that once per year & $27(26 \%)$ \\
\hline Once per year & $17(16 \%)$ \\
\hline 2-4 times per year & $12(12 \%)$ \\
\hline Every other month & $5(5 \%)$ \\
\hline Every month & $11(11 \%)$ \\
\hline \multicolumn{2}{|c|}{$\begin{array}{l}\text { To what extent would you like to be engaged in Global } \\
\text { EM activities in the future? }\end{array}$} \\
\hline Much more & $30(29 \%)$ \\
\hline Little bit more & $43(41 \%)$ \\
\hline About the same & $28(27 \%)$ \\
\hline Little bit less & $2(2 \%)$ \\
\hline Much less & $2(2 \%)$ \\
\hline
\end{tabular}

Results and draft recommendations were presented at the CAEP 2018 Academic Symposium. Feedback from the Academic Section was incorporated into the final recommendations presented here (Box 1).

\section{Limitations}

The general survey had a low response rate and was likely completed by those with an interest in Global EM. 
Therefore, results may not reflect CAEP general membership. The Global EM practitioner surveys were limited to those identified by panelists and their networks. We recognize that many practising Global EM physicians may not have been reached by these methods. Recommendations are targeted to academic centres and may not all be applicable in smaller emergency centres.

It should be noted that these recommendations are not meant to address how individuals engage in Global EM, but rather how Canadian departments/divisions can provide a supportive academic environment that fosters opportunities to engage. The panel recognizes that emphasis should be placed on responsible engagement, including initiatives that are evidence-based, build local capacity, and encourage collaboration with local and international organizations.

\section{RECOMMENDATIONS}

\section{Recommendation 1: Acknowledge that Global EM is a fundamental part of EM and should be included in academic department and division activities}

Striving for excellence in the field of EM should include sharing that expertise in the global setting. Recognition of EM as a unique body of knowledge and expanding emergency care provision and training will be possible only through collaborative global efforts. Global EM practice also includes recognizing and addressing inequalities in emergency care in Canada in remote, vulnerable, and underserved populations. Global EM may include clinical, educational, public health, policy development, disaster response, administrative, or research endeavors. EM departments/divisions must all recognize and accept some responsibility for ameliorating the current inequalities in global emergency care.

\section{Recommendation 2: Academic departments and divisions should develop a strategic plan that allows for the implementation, growth, support, and promotion of Global EM initiatives and activities}

The growth of Global EM requires intentional planning for initial development and implementation, as well as ongoing support and future expansion. Strategic plans ought to define Global EM scope of activities, consider Global EM in the context of the department/division and the broader work environment, set goals and timelines,
Box 1. Summary of recommendations for developing and supporting Global Emergency Medicine in Canadian academic emergency departments and divisions

\section{Academic department and divisions}

- Acknowledge that Global EM is a fundamental part of emergency medicine and should be included in department/division activities.

- Develop a strategic plan that allows for the implementation, growth, support, and promotion of Global EM initiatives and activities.

- Identify a Global EM leader or leaders in their department or seek out leadership to champion the development of Global EM.

- Provide support and foster the development of Global EM. Identify barriers to the practice of Global EM in their department/division and seek solutions.

- Promote and establish collaborations between Global EM faculty and other global health practitioners and organizations.

- Include Global EM topics in educational activities and encourage additional training specific to Global EM.

- Academic deliverables should be an output from Global EM activities, and Global EM activities should be acknowledged as academic accomplishments.

CAEP

- Develop and implement a strategic plan for Global EM engagement in Canada.

- Increase its own engagement in Global EM and develop avenues to support its members in Global EM practice.

and consider how to address challenges. Strategic plans should be written documents that can be reviewed and adapted as both Global EM and the department/division evolves. They may be stand-alone documents, or incorporated into existing departmental/division strategic plans.

\section{Recommendation 3: Academic departments and divisions should identify a Global EM leader or leaders in their department or seek out leadership to champion the development of Global EM}

EM as a specialty was established through passionate leadership, which provided opportunity for growth in a key clinical area, and Global EM practitioners consistently identified quality mentorship as key to their career development. Many EM physicians currently participate in a wide range of global health initiatives. These 
colleagues should be identified, acknowledged, and encouraged to lead the development of Global EM. If no physicians are currently engaged in global health initiatives, academic departments/divisions should consider recruiting those with an interest. Leaders should then participate in strategic plan development, identify local barriers to Global EM practice, foster collaboration with other global health practitioners, and encourage opportunities in the field.

Recommendation 4: Academic department and divisions should provide support and foster the development of Global EM, as well as identify barriers to the practice of Global EM in their department/division and seek solutions

Following identification of Global EM leadership in all departments, other types of support for development of a Global EM program should be identified. Our surveys identified many barriers that need to be addressed (Supplementary Material, Table 3), and individual departments/divisions may identify additional obstacles. Mitigation strategies should be developed for each barrier identified. Barriers may require local solutions, but ideas should be shared between departments.

It is critical to identify dedicated space and time for Global EM programs to flourish. Innovative ways to support Global EM could come from allocated resources of diverse organizations (university programs, governmental vulnerable population programs, Royal College education and international programs, CAEP, and so on). Other examples of support include flexible scheduling options, shared FTEs, including Global EM topics in academic sessions, administrative and research support, and acknowledgement of academic Global EM contributions.

Recommendation 5: Academic departments and divisions should promote and establish collaborations between Global EM faculty and other global health practitioners and organizations

Most universities in Canada have some global initiatives in research, education, or administration with networks to support these areas of global focus. As EM interacts with all medicine programs, it is ideally placed to collaborate with local departments, as well as programs across the country. Shared learning, resources, and networks will enable global health program development, eliminate redundancy, and promote efficiency for initiatives.
Collaborations with medical societies (e.g., IFEM, Wilderness Medical Society, World Organization of Family Doctors, World Association for Disaster and Emergency Medicine, national EM groups) and organizations (e.g., World Health Organization, Médecins Sans Frontières, Médecins du Monde, International Medical Corps, Canadian Coalition for Global Health Research) should be established. These collaborations are beneficial to academic global initiatives, individual practitioners, and the organizations. Most importantly, collaborations help ensure that initiatives are not developed in isolation, but rather work in tandem with organizations, universities, or governments to improve the overall impact.

Recommendation 6: Academic departments and divisions should include Global EM topics in educational activities and encourage additional training specific to Global EM

Global EM topics should be included within regular educational initiatives, including grand rounds, journal clubs, and resident curriculum. For trainees interested in Global EM, additional exposure should be made available through international electives, field placements, and mentoring. For most individuals seeking to include Global EM in their careers, additional training will be warranted covering tropical medicine, humanitarian and disaster response, public health, and/or EM systems, depending on the individual's focus within Global EM.

Recommendation 7: Academic deliverables should be an output from Global EM activities, and Global EM activities should be acknowledged as academic accomplishments

Academic deliverables are key if Global EM is to be a sustainable career path for individuals working in academic departments/divisions. Like other academic domains, deliverables will include publication of peer-reviewed manuscripts, conference presentations, teaching and mentoring, and securing peer-reviewed grant funding. Involvement in Global EM policy-making and advocacy is also important, as is dissemination of academic endeavors and results. Scholarly academic Global EM activities need to be supported by departments/divisions and home institutions. Furthermore, these academic outputs should be recognized as academic accomplishments and contribute to advancement and promotion in the academic environment. 
Recommendation 8: CAEP should develop and implement a strategic plan for Global EM engagement in Canada

CAEP should also develop a strategic plan to help support departments/divisions in developing their Global EM engagement. This would include a CAEP definition of Global EM and position statements regarding Global EM engagement. As CAEP links EM practitioners across the country, it should also be a resource where Canadian Global EM practitioners can build networks for collaborations in Canada and internationally.

\section{Recommendation 9: CAEP should increase its own engagement in Global EM and develop avenues to support its members in Global EM practice}

CAEP's engagement should include continued support of a CAEP wide interest group. The Global Emergency Medicine Academy within the Society of Academic Emergency Medicine (SAEM), as well as the International Emergency Medicine section in the American College of Emergency Physicians (ACEP), provides examples of similar well-established groups. ${ }^{17,18}$ The Global EM section of the CAEP website requires updating to provide links to ongoing initiatives, research, and Global EM resources. There could be increased opportunities for Global EM practitioners to access educational and research funding. Global EM should be included as a track in all future CAEP conferences, and CFEM should increase support for Global EM publications. CAEP could further increase its engagement with higher level Global EM organizations, including IFEM and the WHO, and support member involvement in these international organizations.

\section{CONCLUSION}

We present nine recommendations to increase engagement in Global EM (seven for Canadian academic departments/ divisions, two for CAEP). These recommendations should serve as a starting point for departments and divisions to assess local barriers to Global EM engagement and develop strategic plans to increase involvement in the field. The development and improvement of EM should not be limited to the Canadian context, and all academic departments/divisions striving for excellence should play a role in improving access to emergency care around the world.
Acknowledgements: The authors would like to thank Dr. James Ducharme for reviewing the recommendations and providing his expertise. They would also like to thank Shanna Scarrow and Kelly Wyatt from CAEP for their assistance with organization, panel meetings, survey distribution, and symposium organization; and Amanda Ross-White from the Queen's University Library for helping with the literature search.

Supplementary material: The supplementary material for this article can be found at https://doi.org/10.1017/cem.2019.377.

\section{REFERENCES}

1. Obermeyer Z, Abujaber S, Makar M et al. Emergency care in 59 low- and middle-income countries: a systematic review. Bull World Health Organ 2015;93:577-86.

2. IFEM. IFEM vision and mission statement; 2016. Available at: https://www.ifem.cc/about-us/vision-mission-goals/ (accessed August 16, 2018).

3. VanRooyen M, Venugopal R, Greenough PG. International humanitarian assistance: where do emergency physicians belong? Emerg Med Clin North Am 2005;23(1):115-31.

4. Meshkat N, Misra S, Hunchak C, et al. Knowledge gaps in the diagnosis and management of patients with tropical diseases presenting to Canadian emergency departments: are the gaps being met? CFEM 2014;16(6):458-66.

5. Ohle R, Bleeker H, Yadav K, Perry J. The immigrant effect: factors impacting use of primary and emergency department care - a Canadian population cross-sectional study. CFEM 2018;20(2):260-5.

6. Eggertson L. Canadians respond to Katrina's devastation. CFEM 2005;173(7):743.

7. Tubman M, Maskalyk J, Mackinnon D, et al. Tackling challenges of global health electives: resident experiences of a structured and supervised medicine elective within an existing global health partnership. Can Med Educ 7 2017;8 (2):E4-10.

8. Goldman RD, Cheng A, Jarvis A, et al. An international fellowship training program in pediatric emergency medicine: establishing a new subspecialty in the land of the dragon. Pediatr Emerg Care 2011;27(12):1208-12.

9. Society for Academic Emergency Medicine. Fellowship directory; 2018. Available at: http://www.saem.org/membership/services/fellowship-directory (accessed August 1, 2018).

10. International Emergency Medicine Fellowship Consortium. Programs; 2017. Available at: http://iemfellowships.com/programs/ (accessed August 1, 2018).

11. Dey CC, Grabowski JG, Gebreyes K, et al. Influence of international emergency medicine opportunities on residency program selection. Acad Emerg Med 2002;9(7):679-83.

12. Canadian Association of Emergency Physicians. CAEP Academic Section; 2018. Available at: https://caep.ca/em-community/academic-section/ (accessed August 29, 2018).

13. GEMLR. Consensus definition for global emergency medicine; 2018. Available at: https://www.gemlr.org/discussion. html (accessed August 16, 2018).

14. Martin IB, Levine AC, Kayden S, Hauswald M. More than just a hobby: building an academic career in global emergency medicine. 7 Emerg Med 2014;47(1):107-12. 
15. Hunchak C, Puchalski Ritchie L, Salmon M, et al. LO15: not a hobby anymore: establishment of the Global Health Emergency Medicine organization at the University of Toronto to facilitate academic careers in global health for faculty and residents. CFEM 2017;19(S1):S32.

16. Alagappan K, Schafermeyer R, Holliman CJ, et al. International emergency medicine and the role for academic emergency medicine. Acad Emerg Med 2007;14(5):451-6.
17. American College of Emergency Physicians. International Emergency Medicine Section; 2019. Available at: https:// www.acep.org/how-we-serve/sections/international-emergency-medicine/\#sm.00001le6ppoyb4eiszakrq8c6qftp (accessed August 16, 2018).

18. Global Emergency Medicine Academy. Mission and strategic plan; 2019. Available at: http://www.saem.org/gema/utilitymenu/about (accessed August 16, 2018). 